\title{
Las Nuevas Tecnologías y las Redes Sociales en la Comunicación para la Solidaridad: análisis de una campaña de sensibilización y denuncia en Twitter
}

\author{
Marta Isabel GonzÁLez Álvarez \\ UNIR-Universidad Internacional de la Rioja ${ }^{1}$ \\ martaisabelgonzalez@yahoo.es
}

\begin{abstract}
Resumen
Las Campañas de Denuncia y Sensibilización Social son prácticas comunicativas habituales en las entidades del Tercer Sector o Sector Social, especialmente las ONG, que necesitan comunicar las injusticias sociales y de falta de desarrollo para poder fomentar así cambios de actitudes en las personas que pongan solución a esa realidad denunciada. Las nuevas tecnologías, Internet y desde 2006, las Redes Sociales, son herramientas e instrumentos esenciales para que las organizaciones puedan lograr los objetivos de la Comunicación para la Solidaridad ${ }^{2}$ de manera más rápida, económica, directa y eficaz.
\end{abstract}

Palabras clave: Comunicación para la Solidaridad, Nuevas Tecnologías, Internet, Redes Sociales, Twitter, Campañas de Sensibilización y Denuncia.

New Technologies and Social Networks in Communication for Solidarity: analysis of an awareness campaign and petition on Twitter

\begin{abstract}
Campaigns and Social Awareness Report communicative practices are common in Third Sector and Social Sector, especially NGOs, who need to communicate social injustice and lack of development in order to promote changes in attitudes and people who put solution reported that reality. New technologies, Internet and since 2006, Social Networks, tools and instruments are essential for organizations to achieve the objectives of the Communication for Solidarity, in a way faster, economic, directly and effectively.
\end{abstract}

Key Words: Communication for Solidarity, New Technologies, Social Networks, Communication for Solidarity, Twitter, Awareness Campaign

Referencia normalizada:

González Álvarez, M. I. (2013) Las Nuevas Tecnologías y las Redes Sociales en la Comunicación para la Solidaridad: análisis de una campaña de sensibilización y denuncia en Twitter. Historia y Comunicación Social. Vol. 18. № Especial Noviembre. Págs. 689-701.

Sumario: 1. Introducción. 2. Metodología empleada. 3. De Comunicación para el Desarrollo al nuevo enfoque de la Comunicación para la Solidaridad 4. Las Tecnologías de Información y Comunicación (TIC) y su relación con la Comunicación para la Solidaridad. 5. Uso de las Nuevas Tecnologías en las

1 Miembro de Grupo de Investigación “Comunicación y Sociedad Digital” de la UNIR-Universidad Internacional de la Rioja.

2 El concepto Comunicación para la Solidaridad, es un término propuesto previamente por la autora. (González Álvarez, 2010) 
ONG: ventajas e inconvenientes. 6. Análisis de "Un tuit contra la ablación \#stopablacion" 7. Conclusiones, 8. Bibliografía y 9. Anexo

\section{Introducción}

En las últimas décadas las Campañas de Denuncia y Sensibilización Social se han convertido herramientas habituales de comunicación de las entidades del Tercer Sector, esas asociaciones, fundaciones, cooperativas y, especialmente, de las ONG, o entidades del Tercer Sector sin ánimo de lucro y con objetivos solidarios, en las que se contempla la necesidad de sensibilizar y denunciar las injusticias sociales o de desequilibrio en el nivel de desarrollo humano. En esta línea de trabajo, el avance de las nuevas tecnologías e internet, al que desde el año 2006 se han sumado las redes sociales, se prefiguran como nuevas y eficaces herramientas comunicativas que permiten llegar de manera más económica a un mayor número de personas y de manera más directa.

Partiendo de lo anterior, nos preguntamos ¿cuáles son las ventajas, fortalezas y oportunidades que ofrecen las nuevas tecnologías y cuáles sus desventajas, puntos débiles y posibles amenazas? ¿Ayudan estas nuevas tecnologías a la sensibilización sobre cuestiones sociales o de falta de desarrollo y a lograr la Comunicación para la Solidaridad ${ }^{3}$ ? ¿Qué podemos extraer del análisis de una campaña de sensibilización y denuncia realizada íntegramente a través de Internet y usando las Redes Sociales, especialmente Twitter?

Los objetivos específicos de esta investigación son:

- Revisar la literatura sobre Comunicación para el Desarrollo con el fin de contextualizar la que se ha definido como "Comunicación para la Solidaridad"

- Revisar la literatura sobre Comunicación Social y Nuevas Tecnologías y ofrecer un análisis DAFO (Debilidades, Amenazas, Fortalezas y Oportunidades) sobre las Nuevas Tecnologías en relación a esa "Comunicación para la Solidaridad"

- Analizar las ventajas o inconvenientes que el uso de las TIC suponen para las ONG.

- Analizar una campaña de comunicación, sensibilización, denuncia y captación, realizada por una organización del Tercer Sector y dentro del entorno de la "Comunicación para la Solidaridad", en la que se han usado las Nuevas Tecnologías y las Redes Sociales, especialmente Twitter.

3 En una investigación precedente (Cfr. González Álvarez, 2010) propuse el concepto Comunicación para la Solidaridad, del que aquí haré una breve referencia. 


\section{Metodología empleada}

La metodología utilizada para en esta investigación ha sido de carácter mixto: cualitativa y de campo. Cualitativa, con la revisión bibliográfica conceptual sobre comunicación y comunicación para el desarrollo, la revisión documentos, estudios y análisis publicados sobre el Tercer Sector en España; y el análisis de documentos sobre el avance de las Nuevas Tecnologías y su aplicación en las ONG. Y de campo, porque se ha analizado un caso concreto de campaña de comunicación, sensibilización denuncia y captación: la iniciativa "Un tuit contra la ablación" realizada por la ONG World Vision a través de Internet y de la Red Social Twitter, enmarcada en la campaña "Stop Ablación/ \#StopAblacion” en su edición del año 2013.

\section{De comunicación para el desarrollo al nuevo enfoque de la comunicación para la solidaridad}

La información "revoluciona nuestro modo de vida y debería ser un proceso volcado en proporcionar a los individuos el poder del conocimiento para que tomen decisiones reflexivas con el fin de mejorar sus vidas y el conjunto de la sociedad" (Markiewicz, 2002:187) y la comunicación es "una herramienta que puede provocar la modificación de conductas y el desarrollo político, social y económico" (Basz, 2008:59).

Bernabé define la Comunicación para el Desarrollo como un "campo multidisciplinar de las relaciones internacionales" (Bernabé, 2001:128) que nace del ámbito de la Cooperación al Desarrollo, y que tiene como principales protagonistas entidades y organismos internacionales, los medios de comunicación y medios de Comunicación tanto en el Norte como en el Sur y a ONG (asociaciones, fundaciones,...).

Pero la comunicación tiene una importancia estratégica y "estar informados y comunicados permite extender oportunidades, y que así la comunicación se convierte en una finalidad constitutiva del desarrollo que deseamos promover" (Martínez y Lubetkin, 2008:134) y por eso los países, organizaciones y pueblos del Sur, ya no sólo piden ayuda económica sino también, el derecho a expresarse sin mediación o representantes, a ser escuchados y tenidos en cuenta. Es así como se pasa de la Comunicación "para" el Desarrollo hasta convertirse en una Comunicación "como" Desarrollo ${ }^{4}$, es decir, la comunicación entendida como un objetivo de desarrollo más. Esto ocurre cuando ésta se convierte en algo que "además de lidiar con cifras y recetas se ocupa, fundamentalmente de la gente".

Avanzando en estos conceptos, en una investigación precedente propuse uno nuevo, el de "Comunicación para la Solidaridad" (González Álvarez, 2010), pues considero que éste amplía la influencia de la Comunicación para el Desarrollo y la abre y ofrece

4 Erro, 2007:20 
como herramienta útil a las organizaciones y a los medios, de manera que se aplique a todo el espectro de procesos solidarios que tienen lugar en la sociedad y no sólo a los que tienen relación con el ámbito del Desarrollo. Así la defino como "la comunicación personal y social que logra desencadenar procesos de solidaridad en cada persona y en la sociedad en su conjunto, logrando, con esos procesos, modificar de manera positiva la realidad" González Álvarez (2010:134). Las principales implicaciones de esta definición son:

- Que por medio de la comunicación externa realizada por las ONG y otras organizaciones del Tercer Sector y multiplicada por los medios de comunicación se sensibiliza y cambia la realidad.

- Que a través de la Comunicación para la Solidaridad se generan cambios de actitud que desencadenan la acción solidaria necesaria para lograr objetivos de desarrollo y dignidad human-.

Esta Comunicación para la Solidaridad tiene seis características esenciales ${ }^{5}$ :

- El tacto informativo: cuidando con detalle el tratamiento informativo y comunicativo de aquellos colectivos más vulnerables.

- El afán de veracidad: mostrando la realidad tal cual es, sin sensacionalismo y sin maquillar la realidad para disimular la situación o para que parezca idílica.

- La contextualización: mostrando hechos y causas, además del contexto político, económico, social, climatológico, etc. y las posibles consecuencias.

- La excelencia profesional: ejerciendo periodistas y comunicadores sus conocimientos y ética con responsabilidad dada la sensibilización que desempeñan.

- La creatividad: para lograr llegar y sorprender al receptor, poniendo rostro a la realidad comunicada y ejemplificando tanto la necesidad como su remedio.

- La cesión del protagonismo: dejando espacio, tiempo, imagen y palabra suficientes a los protagonistas, ya sean de países del Sur o de colectivos "sin voz" del Norte.

Por último nos fijaremos en que el mensaje propio de la Comunicación para la Solidaridad es un "mensaje solidario", lo cual se consigue si éste tiene las siguientes características ${ }^{6}$ :

- Universal: porque, al igual que los Derechos Humanos, el verdadero "mensaje solidario" es universal.

- Altruista: con objetivos de información, sensibilización y denuncia ante situaciones y acontecimientos injustos.

- Humanista: basado en el ser humano y en su bien, defendiendo la vida, la paz, la justicia, la igualdad, la libertad, la responsabilidad y la solidaridad.

5 González Álvarez (2010:238)

6 González Álvarez, (2010:243) 
- Revelador. Haciendo ver y reconocer situaciones, personas y países que necesitan de nuestra solidaridad.

- Realista: sin ocultar ni maquillar la realidad.

- Valiente: ejerciendo el derecho a informar, sin callar ante los poderes públicos, gubernamentales, económicos, etc.

- Vehemente: insistente porque sabe que el fin que se persigue, merece la persistencia en la comunicación.

- Complejo: con datos y contextualización.

- Positivo: buscando, incluso en la situación más difícil a denunciar, una solución al problema y alternativas.

- Sensibilizador: incitando a la acción y evitando lo que el escritor Amin Maalouf ha denominado "periodismo emocional" para definir el que persigue que "nos conmovamos instantáneamente por todo sin ocuparnos de forma duradera de nada".

- Posicionador: evitando la indiferencia y planteando valores absolutos imposibles de defender a medias.

- Implicador: a quien lo escucha, le hace tomar una decisión sobre la cuestión y le invita a implicarse

- Ejemplar: porque sirve de ejemplo y provoca su imitación, aún más si lo emite alguien célebre.

- Creativo: con infografías, recursos audiovisuales y otras herramientas que ayudan a difundir.

- Referenciado: con contenidos y referencias de agencias informativas, declaraciones, informes...etc.

- Colorista: contenidos y mensajes diferentes, que suelen aportar reflexión, color y calor a los medios más grises.

\section{Las tecnologías de información y comunicación (tic) y su relación con la comunicación para la solidaridad}

La llamada "Sociedad Red", está compuesta por Internet y otras Tecnologías de la Información y la Comunicación (TIC) que facilitan la conexión entre nodos: "medios digitales que permiten adquirir, procesar, almacenar, distribuir y acceder a información" (Acevedo Ruiz, 2006:8).

Ante estas TIC, se puede tener una visión o actitud "tecnofóbica, tecnocéntrica o indiferente" (Marí Sáez, 2005:85) pero no podemos negar que los descubrimientos que han hecho posible la aparición de las TIC "están conduciéndonos a ritmos vertiginosos de cambio social y económico y a la aparición de nuevas desigualdades" (Gómez, F. 2009:26) y también, que son herramientas estratégicas para lograr promover el desarrollo humano ya que "la información y el conocimiento son recur- 
sos esenciales para el desarrollo tanto individual como colectivo y su papel principal en el desarrollo es el de "empowerment through information"" (Labelle, 2003).

Así, el "Informe del Milenio" de Naciones Unidas consideraba el aprovechamiento de las TIC para el desarrollo como una de las siete prioridades básicas: "Hay que hacer todo lo posible para maximizar el acceso de sus pueblos a las nuevas redes de información" (Annan, 2000). Y es que, "por primera vez en la historia, cantidades sustanciales de información pueden llegar rápidamente a cualquier persona, en cualquier momento y en cualquier lugar" (Acevedo Ruiz, 2006:9).

López Lita define este nuevo reto y difícil equilibrio entre Nuevas Tecnologías y TIC y la solidaridad y el desarrollo así:

"Fuerzas opuestas luchan por prevalecer: por un lado la vertiginosa velocidad a la que se desarrollan las nuevas tecnologías (...); por otro, las crecientes desigualdades que en función del uso que pueda hacerse de ellas pueden tender a reducirse o a incrementarse, introduciéndonos en una peligrosa dinámica de injusticia y consecuentemente de polarización entre el centro y la periferia, que a medio plazo haría detonar todo el sistema" .

Si además nos fijamos en los medios de comunicación, la llegada de Internet y su uso de manera generalizada en España a partir de mediados de la década de 1990, ha hecho que se genere un nuevo escenario o "ecosistema mediático" (Tascón y Quintana, 2012:84).

A todo esto, se suma que con la Red, muchos periodistas han asegurado que Internet "ha modificado de tal manera la información que supera la censura, la desinformación, la mentira oficializada y otras lacras que pesan sobre ella" (Gourevitch, 2002:113) y que con una cámara o un móvil de última generación en el bolsillo pueda publicar contenidos y noticias al instante, opinar, compartir en redes, etc. Lo que Orihuela (1997 y 1999) ha definido como "prosumidores": "consumidores" y "productores" de informaciones y contenidos.

En este escenario, las Redes Sociales, han logrado el desarrollo peculiar, mucho más con el denominando "ciberactivismo", que no tiene por qué estar usado únicamente como técnicas hacker agresivas como las que enumera Tascón y Quintana (2012:229) sino también como una posibilidad de activismo social y solidario a través de las TIC, Internet y las Redes Sociales.

\section{Uso de las nuevas tecnologías en las ong: ventajas e inconvenientes}

Partimos de que las TIC son una oportunidad de fomento de la Comunicación para la Solidaridad pero siempre que, como asegura Ballesteros éstas se conciban:

7 Empoderamiento a través de la información 
"acompañadas de una reflexión, una apuesta política clara y un estilo comunica-
tivo apropiado para que el potencial de las nuevas tecnologías se oriente a liberar al
individuo y a la comunidad de sus inconvenientes y barreras culturales, políticas y
económicas, evitando que sirvan de acicate a nuevos totalitarismos y desigualdades".
ii

Y es que, citando a Gómez Ferri,

"los nuevos medios de comunicación (...), entre los que destaca Internet, han facilitado que los individuos se interconecten y pierdan el sentimiento de impotencia del que nos hablaba Eco (1963:82), haciendo que la distancia ya no sea óbice ni para organizarse ni para actuar a escala mundial" iii.

Así, "la solidaridad y la cooperación internacionales han dejado de ser asunto exclusivo de las agendas de los organismos nacionales y multilaterales para estar a la orden del día para cualquier ciudadano, por no decir las organizaciones de la sociedad civil” (Ariño, 1999 y 2001).

En este escenario general se sitúan las ONGD españolas, para quienes estas herramientas son fundamentales en el desarrollo de su trabajo por, para y en los Países del Sur, (CONGDE: 2008:45) De hecho, según la misma fuente, ya en 2008 el 99\% de las ONGD disponía de página web que se actualizan periódicamente, casi la mitad (44\%) hace uso de espacios de participación virtuales (foros, blogs, redes sociales), el $28 \%$ usa las videoconferencias de manera habitual y en torno al $40 \%$ dispone de Intranet propia para uso del personal laboral, miembros del órgano de Gobierno y, en algunos casos, también para su base social (socios/as, voluntariado).

Así "la flexibilidad, la horizontalidad, la interconexión y la cercanía" (Marí Sáez, 2005:81) son "fortalezas" o ventajas intrínsecas de la comunicación on-line y de Internet para las organizaciones del Tercer Sector. Y entre las oportunidades que ofrece Internet a los directores de comunicación, destacamos ahora las que tienen que ver con la Comunicación Externa, con ejemplos como las salas de prensa virtuales con recursos gráficos, sonoros, audiovisuales...etc.; el envío masivo de notas de prensa digitales, las entrevistas on-line y las retransmisiones en streaming o tiempo real en directo y también con el monitoreo y seguimiento de la repercusión en medios digitales

Pero además de lo dicho, las nuevas tecnologías pueden plantear ciertas amenazas y debilidades, sobe todo dentro de las organizaciones y no sólo en su comunicación, como por ejemplo, la falta de dominio de "lenguaje electrónico", la "brecha digital interna" o la incapacidad para asumir con suficiente rapidez los avances y la necesidad de nuevos roles que se dan como consecuencia de la implantación de las TIC en las instituciones y en sus departamentos de Comunicación. 


\section{Análisis de "un tuit contra la ablación \#stopablacion"}

Analizar el uso de las Nuevas Tecnologías y las Redes Sociales en la Comunicación para la Solidaridad a través de Campañas de Denuncia y Sensibilización Social no es una cuestión sencilla. Los pocos años transcurridos desde el nacimiento de Facebook (2004), la red social más conocida y usada (mil millones de usuarios registrados en octubre de 2012) o de Twitter, (2006) la red social de microblogging más conocida y usada en la actualidad (500 millones de usuarios en abril de 2012) unido a la velocidad con la que éstas herramientas de comunicación se han propagado, dificulta en ocasiones el análisis científico de estos procesos.

Centrándonos en el caso de la campaña "Un tuit contra la ablación" realizado por la ONG World Vision y enmarcada en su iniciativa "\#StopAblacion", comenzaremos por explicar que la idea surgió porque se detectó una sensibilidad especial de la población española sobre la práctica de la ablación o Mutilación Genital Femenina (MFG) y al existir el Día Mundial de Tolerancia Cero a la Ablación o Mutilación Genital Femenina que se celebra el 6 de febrero, desde los Departamentos de Comunicación, Marketing on-line y Proyectos de la organización se propuso que sería un buen tema para comunicar y un momento oportuno y un buen momento para hacer una campaña así para resaltar el problema y los proyectos que realiza la organización para luchar contra esa práctica, además de recaudar fondos privados, ya que en World Vision los proyectos que luchan contra esa situación se realizan sin ningún tipo de subvención pública.

Para comprender mejor la iniciativa de 2013 "Un tuit contra la ablación" hay que comprender lo sucedido el año anterior. En 2012 World Vision había realizado dos acciones off line: un cine fórum con expertos sobre la cuestión: antropóloga, abogada, mujer africana que trabaja con población inmigrante en España... y una acción de calle o Street marketing con la que se repartían flore con un flyer o folleto explicativo a cambio de un donativo. Estas acciones fueron combinadas con una iniciativa en las redes sociales, que ya tenían abiertas en la organización desde el segundo semestre de 2008 y en ese año decidieron hacer la iniciativa "6 tuits por la ablación" tras el 6 de febrero. Para ello buscaron a mujeres usuarias de Twitter (Tuiteras) que se seleccionaron según tres criterios: que fueran muy activas, que tuvieran un Blog u otro soporte desde el que informar o que en algún momento hubieran dialogado en redes sociales sobre temas de género. Se les envió un DM (mensaje directo privado a través de Twitter) y les propusieron unirse a la iniciativa. Hicieron un mailing y una web a través de la que poder hacer donativos (landing page). En esta ocasión y pese a que la web de la campaña fue hackeada, el día 6 de febrero fue la tercera más visitada, el hashtag (tema de interés) de la campaña \#stopablacion fue TT (trending topic, tema más difundido en un periodo de tiempo concreto) en Barcelona durante el día 6 de febrero. Además, y aunque el objetivo no era ese, aumentaron de seguidores en Twitter y Facebook .

Tras la experiencia tan buena de 2012, 6 meses antes del 6 de febrero de 2013, en World Vision ya comenzaron a preparar la campaña y decidieron que en esta ocasión 
sería $100 \%$ online y con Twitter. Se creó la web www.stopablacion.com que insertada en la web de la organización http://www.worldvision.es/stopablacion permite elegir entre 2 opciones generadas a través de un juego de palabras de la palabra "ablación":

- "hABLA" con las siguientes secciones: Qué es la ablación; Así es posible evitarla; Historias de éxito y Quiénes somos (con información básica de la $\mathrm{ONG})$

- “acCIÓN" con tres secciones generales para facilitar la captación económica: Tuiteras contra la ablación; Apadrina niñas para luchar contra la ablación y Donar para luchar contra la ablación

Para la sección "Tuiteras contra la ablación" se eligieron a las tuiteras Isabel Llano (@isasaweis), Mabel Lozano (@LozanoMabel)y la Red Social de Madres CharHadas (@CharHadas).Rosana Guiza@RosanaGuiza)y Ana Milán (@_AnaMilan_ ) Con todas ellas la ONG ya había trabajado en diferentes circunstancias. Además eran personas conocidas, con muchos seguidores, actrices...Les propusieron la idea y hacer tuits con \#StopAblacion en torno al día internacional y sobre todo el 6 de febrero. Les enviaron Kits de la campaña para que lo hicieran con información. Esta colaboración fue gratuita y cada una publicaba lo que podía.

Pero además de ser una iniciativa de comunicación, sensibilización y denuncia, es una iniciativa de captación. Por eso cada una de estas "tuiteras" era una especie de "embajadora de la organización" con objetivos de "recaudación" de donativos concretos (a través de SMS solidarios) y esto aporta a la campaña además mucha transparencia informativa y de cuentas, ya que a través de la web en su parte "Acción" http://www.worldvision.es/stopablacion/accion/ se puede ver exactamente cuánto se lleva recaudado en cada uno de los equipos y qué objetivos de recaudación tienen a lo largo de todo el año 2013. Además de estar todo apoyado también a través de Google Adwords (anuncios por palabras que aparecen en los buscadores de Google), en su versión Google Grants (gratis para las ONG).

Lo más significativo y lo que más salió también en los medios de comunicación a través de las Notas de Prensa, es que ha sido una campaña sin presupuesto, que:

- Por primera vez se ha realizado sólo online

- Por primera vez sólo a través de Twitter

- Por primera vez con "tuiteras" recaudando para la organización.

Los objetivos principales de la campaña eran recaudatorios para seguir luchando contra la ablación en los países en los que la ONG actúa. Pero además, como resultados la organización explica que:

- Aumentaron en un 20\% los seguidores de Twitter (y un 3\% en Facebook).

- Ganaron en Twitter unos 15 "influencers" o personas de prestigio y con muchos seguidores que son más influyentes a la hora de difundir mensajes (Rosa Montero, Rossy de Palma...). 
- Tuvieron 78 impactos en medios de comunicación (prensa, radio, TV y online)

- Además, las cifras de recaudación a través de SMS siguen subiendo y en todas las opciones que facilitan en la sección "acCION" de la web, siguen recaudando. Y lo curioso es que aunque la iniciativa ha sido sólo online, hay grupos de estudiantes, empresas y otros particulares que están generando eventos para recaudar fondos para la campaña.

Desde la organización no dudan en explicar que las redes y los medios online son más adecuados para generar la "acción" (y en este caso animar a la donación) pero que es cierto que la campaña ha estado construida para explicar más la problemática y sensibilizar y que la repercusión hace que la gente conozca mejor en qué consiste la ablación y quede mejor explicada.

\section{Conclusiones}

Tras lo visto hasta aquí podemos concluir que las Nuevas Tecnologías y las Redes Sociales son actualmente y en el "ecosistema mediático" en el que nos movemos, una herramienta indispensable para lograr poner en práctica la Comunicación para la Solidaridad.

Teniendo en cuenta que la Comunicación para la Solidaridad es "la comunicación personal y social que logra desencadenar procesos de solidaridad en cada persona y en la sociedad en su conjunto, logrando, con esos procesos, modificar de manera positiva la realidad" (González Álvarez, 2010:134) parece claro que cuando se inicia una acción comunicativa usando medios online y redes sociales que logran llegar a las personas de manera muy directa y emitimos un mensaje claro y sensibilizador que propone la acción, los resultados son los que la Comunicación para la Solidaridad propone

\section{Bibliografía}

Libros

ACEVEDO RUIZ, M. (2006), Integración de las tecnologías de la información y comunicación. Asignatura pendiente de la cooperación, CONGDE, Madrid.

ANNAN, K. (2000), We the People: The Role of the United Nations in the $21^{\text {st }}$ Century: Millenium Report, United Nations Dept. of Public Information, New York.

ARIÑO, A. (dir. Et alt) (1999), La rosa de las solidaridades. Necesidades sociales y voluntariado en la Comunidad Valenciana, Fundación Bancaixa. Valencia. 
ARIÑO, A. (dir. Et alt) (2001), La ciudadanía solidaria, Fundación Bancaixa. Valencia.

BENET, V.J. y NOS ALDÁS, E. (eds.) (2003), La publicidad en el Tercer Sector. Tendencias y perspectivas de la comunicación solidaria. Icaria. Barcelona.

COORDINADORA DE ONG PARA EL DESARROLLO-ESPAÑA (CONGDE) (2008), Informe de la CONGDE sobre el sector de las ONGD 2008 edita CONGDE-Coordinadora de ONG para el Desarrollo - España. Madrid.

COORDINADORA DE ONG PARA EL DESARROLLO-ESPAÑA (CONGDE) (2008b), Informe de la CONGDE sobre el sector de las ONGD 2007. Especial temático: base social y participación ciudadana en las ONGD, edita CONGDE-Coordinadora de ONG para el Desarrollo - España. Madrid.

COORDINADORA DE ONG PARA EL DESARROLLO-ESPAÑA (CONGDE) (2010) Informe de la Coordinadora de ONG para el Desarrollo -España sobre el sector de las ONGD en 2009, edita CONGDE-Coordinadora de ONG para el Desarrollo - España. Madrid.

GONZÁLEZ ÁLVAREZ, M. I. (2010), Comunicación para la Solidaridad: las ONG y el papel de la comunicación social y periodística en la sensibilización de la opinión pública y el desarrollo Tesis Doctoral inédita disponible en http://eprints. ucm.es/11633

LABELLE, R. (2003), Information and Communication Technologies (ICTs) for Development in National Human Development Reports, Informe interno para PNUD disponible en http://unctad.org/en/Docs/iteipc20031_en.pdf

MARTÍNEZ, R. y LUBETKIN, M. (eds.) (2008), Comunicación y desarrollo: en busca de la coherencia, Fundación Carolina y Siglo XXI de España Editores S.A. Madrid.

NOS ALDÁS, E. (Ed.) (2002) Medios periodísticos, cooperación y acción humanitaria. ¿Relaciones imposibles? Icaria. Barcelona.

ORIHUELA, José Luis y GARCÍA IRIARTE, Iranzu. (1997) Potencial comunicativo de las redes hipermedia de información distribuida editado por Mundo Internet 97, Asociación de Usuarios de Internet, Madrid.

ORIHUELA, José Luis y GARCÍA IRIARTE, Iranzu. (1997), Periodismo en la Red. En busca del paraíso digital. Expo@Internet 97, Asociación de Usuarios de Internet, Madrid.

ORIHUELA, José Luis y SANTOS, $\mathrm{M}^{\mathrm{a}}$ Luisa. (1999), Introducción al diseño digital. Concepción y desarrollo de proyectos de comunicación interactiva. Anaya Multimedia, Madrid.

TASCÓN, M. Y QUINTANA, Y. (2012) Ciberactivismo. Las nuevas revoluciones de las multitudes conectadas, Ediciones de la Catarata. Madrid

VV.AA. (2005) Solidaridad en red. Nuevas tecnologías, ciudadanía y cambio social Ed. HEGOA, (INSTITUTO DE ESTUDIOS SOBRE DESARROLLO Y COOPERACIÓN INTERNACIONAL-UNIVERSIDAD DEL PAÍS VASCO) Bilbao. 
Capítulos o artículos en libros o revistas en papel

BALLESTEROS CARRASCO, B. (2005), La Comunicación para el Desarrollo: Concepto y problemas de aplicación en la Cooperación Española, en Revista Española de Desarrollo y Cooperación, número 15, Otoño/Invierno 2005. Ed. Instituto Universitario de Desarrollo y Cooperación (IUDC), Universidad Complutense de Madrid. Madrid.

BASZ, P. (2008), Las dificultades comunicativas de las agencias de desarrollo en MARTÍNEZ, R. y LUBETKIN, M. (eds.), Comunicación y desarrollo: en busca de la coherencia. Madrid: Fundación Carolina y Siglo XXI de España Editores S.A. Madrid.

BERNABÉ FRAGUAS, J. (2001), Las áreas de comunicación de las ONGD y el trabajo periodístico en Revista Española de Desarrollo y Cooperación, 7, Otoño/ Invierno .

ERRO SALA, J. (2007), La ayuda humanitaria y los medios: ¿relaciones conflictivas y/o complicidad encubierta? Apuntes para un debate político en ARCAS MANTAS, Irene (2007) Medios de comunicación y organizaciones humanitarias en la respuesta a las crisis editado por y que es resumen de las Jornadas homónimas organizadas por el IECAH (Instituto de Estudios sobre Conflictos y Acción Humanitaria)coordinadas por REY MARCOS, F. NÚÑEZ VILLAVERDE, J. A. y GARCÍA CALERO, F. y celebradas en La Casa Encendida en noviembre de 2006. Madrid.

GÓMEZ, F. (2009), La brecha digital: la nueva era de las desigualdades. La revolución de las Tecnologías de la Información y la Comunicación y su efecto en el desarrollo, en Revista Intervida, no 37, Pags. 26-30, Diciembre, Barcelona.

GÓMEZ FERRI, J. (2003), Sensibilizando a los otros, solidarizándonos con ellos: la antropología en la intervención social solidaria en BENET, V.J. y NOS ALDÁS, E. (eds.) (2003), La publicidad en el Tercer Sector. Tendencias y perspectivas de la comunicación solidaria. Icaria. Barcelona.

GOUREVITCH, A. (2002), Globalización, comunicación e información, en NOS ALDÁS, E. (Ed.) (2002) Medios periodísticos, cooperación y acción humanitaria. ¿Relaciones imposibles? Icaria. Barcelona.

LÓPEZ LITA, R. (2002), El debate sobre el término globalización, en NOS ALDÁS, E. (Ed.) (2002) Medios periodísticos, cooperación y acción humanitaria. ¿Relaciones imposibles? Icaria. Barcelona.

MARÍ SÁEZ, V. M. (2005), Nuevas tecnologías de la información, movimientos sociales y cambio social, en VV.AA. (2005), Solidaridad en red. Nuevas tecnologías, ciudadanía y cambio social Ed. HEGOA, (INSTITUTO DE ESTUDIOS SOBRE DESARROLLO Y COOPERACIÓN INTERNACIONAL-UNIVERSIDAD DEL PAÍS VASCO), Bilbao.

MARKIEWICZ, E. (2002), Dándole utilidad social a la in formación, en NOS ALDÁS, E. (Ed.) (2002) Medios periodísticos, cooperación y acción humanitaria. ¿Relaciones imposibles? Icaria. Barcelona.

McMILLAN, D. \& CHAVIS, D. (1986). Sense of community: a definition and theory en Journal of Community Psychology, 14 
SÁDABA, I. (2005), Del cambio tecnológico al cambio social. Conflictos y protestas globales en la red, en V.AA. (2005), Solidaridad en red. Nuevas tecnologías, ciudadanía y cambio social Ed. HEGOA, (INSTITUTO DE ESTUDIOS SOBRE DESARROLLO Y COOPERACIÓN INTERNACIONAL-UNIVERSIDAD DEL PAÍS VASCO), Bilbao

\section{Notas}

i López Lita, 2002, p.106

ii Ballesteros, 2005, p.13

iii Gómez Ferri, 2003, p. 154

\section{La autora}

Marta Isabel González Álvarez [Astorga (León) 1975] es Doctora en Ciencias de la Información por la Universidad Complutense de Madrid (UCM), Curso Superior en Comunicación y Nuevas Tecnologías (IES-CEU- San Pablo), Técnico en Comunicación Integral por la Universidad Francisco de Vitoria (UFV) y Licenciada en Ciencias de la Información-Periodismo por la UFV y la UCM. Periodista con experiencia en Radio, TV, Prensa Escrita y Digital, compatibiliza desde el año 2000 su trabajo como Directora de Comunicación y Jefa de Prensa en entidades del Tercer Sector con la docencia universitaria y la investigación científica. Es miembro del Grupo de Investigación "Comunicación y Sociedad Digital" de la UNIR, docente y ponente habitual en Másters, Mesas Redondas, Seminarios y Jornadas y experta en Consultoría de Comunicación Institucional, Comunicación, Sensibilización y Marketing Social, Planificación de Comunicación y Comunicación On-Line, Arquitectura Web y Community Strategist. 